Article

\title{
Qualitative Behavior of Unbounded Solutions of Neutral Differential Equations of Third-Order
}

\author{
M. Sathish Kumar ${ }^{1,+} \mathbb{D}$, R. Elayaraja ${ }^{2,+}$, V. Ganesan ${ }^{3,+}$, Omar Bazighifan ${ }^{4,5,+} \mathbb{D}$, Khalifa Al-Shaqsi ${ }^{6,+}$ \\ and Kamsing Nonlaopon $7, *,+\mathbb{D}$
}

1 Department of Mathematics, Paavai Engineering College (Autonomous), Namakkal 637 018, Tamil Nadu, India; msksjv@gmail.com

2 Department of Mathematics, Annai Mathammal Sheela Engineering College, Namakkal 637 013, Tamil Nadu, India; elaya.ams@gmail.com

3 PG and Research Department of Mathematics, Aringar Anna Government Arts College, Namakkal 637 002, Tamil Nadu, India; ganesan_vgp@rediffmail.com

4 Section of Mathematics, International Telematic University Uninettuno, Corso Vittorio Emanuele II, 39, 00186 Rome, Italy; o.bazighifan@gmail.com

5 Department of Mathematics, Faculty of Science, Hadhramout University, Hadhramout 50512, Yemen

6 Department of Information Technology, Nizwa College of Technology, University of Technology and Applied Science, P.O. Box 75, Nizwa 612, Oman; khalifa.alshaqsi@nct.edu.om

7 Department of Mathematics, Khon Kaen University, Khon Kaen 40002, Thailand

* Correspondence: nkamsi@kku.ac.th; Tel.: +668-6642-1582

+ These authors contributed equally to this work.

check for updates

Citation: Kumar, M.S.; Elayaraja, R.; Ganesan, V.; Bazighifan, O.;

Al-Shaqsi, K.; Nonlaopon, K.

Qualitative Behavior of Unbounded

Solutions of Neutral Differential

Equations of Third-Order. Fractal

Fract. 2021, 5, 95. https://doi.org/

10.3390 / fractalfract5030095

Academic Editors: Arran Fernandez and Burcu Gürbüz

Received: 8 July 2021

Accepted: 6 August 2021

Published: 12 August 2021

Publisher's Note: MDPI stays neutral with regard to jurisdictional claims in published maps and institutional affiliations.

Copyright: (c) 2021 by the authors. Licensee MDPI, Basel, Switzerland. This article is an open access article distributed under the terms and conditions of the Creative Commons Attribution (CC BY) license (https:/ / creativecommons.org/licenses/by/ $4.0 /)$.

\begin{abstract}
New oscillatory properties for the oscillation of unbounded solutions to a class of thirdorder neutral differential equations with several deviating arguments are established. Several oscillation results are established by using generalized Riccati transformation and a integral average technique under the case of unbounded neutral coefficients. Examples are given to prove the significance of new theorems.
\end{abstract}

Keywords: neutral differential equation; oscillation; asymptotic behavior; deviating arguments

\section{Introduction}

In this work, we investigate the oscillation properties of solutions to the third-order neutral differential equations with several deviating arguments

$$
\left(r(\iota)\left(z^{\prime \prime}(\iota)\right)^{\alpha}\right)^{\prime}+\sum_{i=1}^{n} q_{i}(\iota) x^{\alpha}\left(\phi_{i}(\iota)\right)=0, \quad \iota \geq \iota_{0}>0,
$$

where $z(\iota)=x(\iota)+p(\iota) x(\varrho(\iota))$ and $\alpha$ is a quotient of odd positive integers.

The main results of this paper are obtained considering the following conditions:

$\left\{\begin{array}{l}r \in C\left(\left[\iota_{0}, \infty\right),(0, \infty)\right) \text { and } \int_{\iota_{0}}^{\infty} r^{-1 / \alpha}(s) d s=\infty ; \\ q_{i}(\iota) \in C\left(\left[\iota_{0}, \infty\right),[0, \infty)\right), \phi_{i}(\iota) \in C\left(\left[\iota_{0}, \infty\right), \mathbb{R}\right) \text { and } \lim _{\iota \rightarrow \infty} \phi_{i}(\iota)=\infty, \text { where } i=1,2, \cdots n ; \\ \varrho \in C\left(\left[\iota_{0}, \infty\right), \mathbb{R}\right) \text { is strictly increasing, } \varrho(\iota)<\iota, \text { and } \lim _{\iota \rightarrow \infty} \varrho(\iota)=\infty ; \\ p(\iota) \in C\left(\left[\iota_{0}, \infty\right), \mathbb{R}\right) \text { with } p(\iota) \geq 1, \text { and } p(\iota) \neq \equiv 1 \text {, eventually. }\end{array}\right.$

By a solution of (1), we mean a function $x:\left[\iota_{x}, \infty\right) \rightarrow \mathbb{R}$ such that $z(\iota) \in C^{2}\left(\left[\iota_{x}, \infty\right), \mathbb{R}\right)$ and $r(\iota)\left(z^{\prime \prime}(\iota)\right)^{\alpha} \in C^{1}\left(\left[\iota_{x}, \infty\right), \mathbb{R}\right)$, and which satisfies Equation (1) on $\left[\iota_{x}, \infty\right)$. We only consider those solutions $x(\iota))$ of (1) defined on some ray $\left[\iota_{x}, \infty\right)$, for some $\iota_{x} \geq \iota_{0}$, which satisfy $\sup \{|x(\iota)|: \iota \geq T\}>0$ for every $T \geq \iota_{x}$. We start with the assumption that Equation (1) does possess a proper solution. A proper solution of (1) is called oscillatory if it has a sequence of large zeros lending to $\infty$; otherwise we call nonoscillatory. 
Because of the enormous advantage of neutral differential equations in describing several neutral phenomena, there is great scientific and academic value in studying neutral differential equations, both theoretically and practically; see [1]. Lately, there have been numerous articles investigating the oscillation of the solutions of third/higher order neutral differential equations with/without deviating arguments; see [2-16].

Baculíková et al. [17], Džurina et al. [18], and Li et al. [19] investigated third-order equations of the form:

$$
\left[a(\iota)\left[x(\iota)+p(\iota) x(\delta(\iota))^{\prime \prime}\right]^{\gamma}\right]^{\prime}+q(\iota) x^{\gamma}(\tau(\iota))=0, \quad \iota \geq \iota_{0} .
$$

Jiang et al. [20] obtained several oscillation results for the third-order equation

$$
\left[a(\iota)\left[x(\iota)+p(\iota) x(\delta(\iota))^{\prime \prime}\right]^{\alpha}\right]^{\prime}+q(\iota) f(x(\tau(\iota)))=0, \quad \iota \geq \iota_{0} .
$$

Tunç [21] investigated the third-order equation

$$
\left(r(\iota)\left((x(\iota)+p(\iota) x(\tau(\iota)))^{\prime \prime}\right)^{\alpha}\right)^{\prime}+\int_{a}^{b} q(\iota, \xi) x^{\alpha}(\phi(\iota, \xi)) d \xi=0 .
$$

Soliman et al. [22] investigated a third-order delay differential equation

$$
\left(a(\iota)\left(\left(x(\iota) \pm \sum_{i=1}^{n} p_{i}(\iota) x\left(\sigma_{i}(\iota)\right)\right)^{\prime \prime}\right)^{\alpha}\right)^{\prime}+\sum_{j=1}^{m} f_{j}\left(\iota, x\left(\tau_{j}(\iota)\right)\right)=0 .
$$

The articles listed above deal with the case when the neutral coefficient $p(\iota)$ is bounded, i.e., the cases where $0 \leq p(\iota) \leq p_{0}<1,-1<p_{0} \leq p(\iota) \leq 0$, and $0 \leq p(\iota) \leq p_{0}<\infty$ were considered, and so the results established in these papers cannot be applied to the case of $p(\iota) \rightarrow \infty$ as $\iota \rightarrow \infty$.

More precisely, the existing literature does not provide any criteria for the oscillation of third-order unbounded neutral differential equations with several deviating arguments in the case when $p(\iota) \rightarrow \infty$ as $\iota \rightarrow \infty$. With this motivation, we provide several criteria for oscillation of the differential Equation (1) under the assumptions of $\varrho(\iota) \geq \phi_{i}(\iota)$ and $\varrho(\iota) \leq \phi_{i}(\iota)$ for $i=1,2, \cdots, n$ when $p(\iota) \geq 1$. Furthermore, the results presented in this paper can be simply extended to more general third-order unbounded neutral differential equations with several deviating arguments in order to achieve more generalized oscillation results. As a result, it is envisaged that the present paper will make a significant contribution to the study of oscillations of solutions of (1).

\section{Main Results}

We start with the following lemmas, which are required to prove our main theorems. Through this paper, we will be using the following notations:

$$
\begin{gathered}
\zeta_{+}^{\prime}(\iota):=\max \left\{0, \zeta^{\prime}(\iota)\right\}, \\
B_{1}\left(\iota, \iota_{1}\right):=\int_{\iota_{1}}^{\iota} \frac{d s}{r^{1 / \alpha}(s)} \text { for } \iota \geq \iota_{1}, \\
B_{2}\left(\iota, \iota_{2}\right):=\int_{\iota_{2}}^{\iota} B_{1}\left(s, \iota_{1}\right) d s \text { for } \iota \geq \iota_{2}>\iota_{1} .
\end{gathered}
$$

Furthermore, throughout this paper, we assume that

$$
\psi_{1}(\iota):=\frac{1}{p\left(\varrho^{-1}(\iota)\right)}\left[1-\frac{1}{p\left(\varrho^{-1}\left(\varrho^{-1}(\iota)\right)\right)}\right]>0
$$


and

$$
\psi_{2}(\iota):=\frac{1}{p\left(\varrho^{-1}(\iota)\right)}\left[1-\frac{1}{p\left(\varrho^{-1}\left(\varrho^{-1}(\iota)\right)\right)} \frac{B_{2}\left(\varrho^{-1}\left(\varrho^{-1}(\iota)\right), \iota_{2}\right)}{B_{2}\left(\varrho^{-1}(\iota), \iota_{2}\right)}\right]>0,
$$

for all sufficiently large $\iota$, where $\varrho^{-1}$ is the inverse function of $\varrho$, and we consider

$$
\Omega_{1}(\iota):=\sum_{i=1}^{n} q_{i}(\iota)\left(\psi_{1}\left(\phi_{i}(\iota)\right)\right)^{\alpha}, \quad \Omega_{2}(\iota):=\sum_{i=1}^{n} q_{i}(\iota)\left(\psi_{2}\left(\phi_{i}(\iota)\right)\right)^{\alpha} .
$$

Lemma 1 ([23]). If $X$ and $Y$ are nonnegative and $\lambda>1$, then

$$
X^{\lambda}-\lambda X Y^{\lambda-1}+(\lambda-1) Y^{\lambda} \geq 0
$$

Lemma 2. If $x(\iota)$ is an eventually positive solution of (1), then $z(\iota)$ satisfies either

$\left(C_{I}\right) z(\iota)>0, z^{\prime}(\iota)>0, z^{\prime \prime}(\iota)>0$, and $\left(r(\iota)\left(z^{\prime \prime}(\iota)\right)^{\alpha}\right)^{\prime} \leq 0$, or

$\left(C_{I I}\right) z(\iota)>0, z^{\prime}(\iota)<0, z^{\prime \prime}(\iota)>0$, and $\left(r(\iota)\left(z^{\prime \prime}(\iota)\right)^{\alpha}\right)^{\prime} \leq 0$.

The proof of the above lemma is standard and thus omitted.

Lemma 3. Let (2) hold, and let $x(\iota)$ be an eventually positive solution of (1) with $z(\iota)$ satisfying $\left(C_{I I}\right)$ of Lemma 2. If

$$
\int_{\iota_{0}}^{\infty} \int_{v}^{\infty} \frac{1}{r^{1 / \alpha}(u)}\left(\int_{u}^{\infty} \Omega_{1}(s) d s\right)^{1 / \alpha} d u d v=\infty
$$

then $\lim _{\iota \rightarrow \infty} x(\iota)=0$.

Proof. Let $x(\iota)$ be an eventually positive solution of (1). Then, there exists $\iota_{1} \in\left[\iota_{0}, \infty\right)$ such that, for $\iota \geq \iota_{1}, x(\iota)>0, x(\varrho(\iota))>0, x\left(\phi_{i}(\iota)\right)>0$ and $i=1,2, \cdots, n$. From the definition of $z$, we have (see also [2] [(8.6)]):

$$
\begin{aligned}
x(\iota) & =\frac{1}{p\left(\varrho^{-1}(\iota)\right)}\left(z\left(\varrho^{-1}(\iota)\right)-x\left(\varrho^{-1}(\iota)\right)\right) \\
& =\frac{z\left(\varrho^{-1}(\iota)\right)}{p\left(\varrho^{-1}(\iota)\right)}-\frac{1}{p\left(\varrho^{-1}(\iota)\right) p\left(\varrho^{-1}\left(\varrho^{-1}(\iota)\right)\right)}\left(z\left(\varrho^{-1}\left(\varrho^{-1}(\iota)\right)\right)-x\left(\varrho^{-1}\left(\varrho^{-1}(\iota)\right)\right)\right) \\
& \geq \frac{z\left(\varrho^{-1}(\iota)\right)}{p\left(\varrho^{-1}(\iota)\right)}-\frac{1}{p\left(\varrho^{-1}(\iota)\right) p\left(\varrho^{-1}\left(\varrho^{-1}(\iota)\right)\right)} z\left(\varrho^{-1}\left(\varrho^{-1}(\iota)\right)\right) .
\end{aligned}
$$

From $\varrho(\iota)<\iota$, (iv) and the fact that $z(\iota)$ is decreasing, we have

$$
z\left(\varrho^{-1}(\iota)\right) \geq z\left(\varrho^{-1}\left(\varrho^{-1}(\iota)\right)\right)
$$

using this in (5), we obtain

$$
x(\iota) \geq \psi_{1}(\iota) z\left(\varrho^{-1}(\iota)\right)
$$

so

$$
x\left(\phi_{i}(\iota)\right) \geq \psi_{1}\left(\phi_{i}(\iota)\right) z\left(\varrho^{-1}\left(\phi_{i}(\iota)\right)\right), \quad i=1,2, \cdots, n
$$

for $\iota \geq \iota_{2}$. Using (6) in (1) gives

$$
\left(r(\iota)\left(z^{\prime \prime}(\iota)\right)^{\alpha}\right)^{\prime}+\sum_{i=1}^{n} q_{i}(\iota)\left(\psi_{1}\left(\phi_{i}(\iota)\right)\right)^{\alpha} z^{\alpha}\left(\varrho^{-1}\left(\phi_{i}(\iota)\right)\right) \leq 0,
$$


for $\iota \geq \iota_{2}$. From (iv)-(v) and the fact that $z(\iota)$ is decreasing, (7) yields

$$
\left.\left(r(\iota)\left(z^{\prime \prime}(\iota)\right)^{\alpha}\right)^{\prime}+z^{\alpha}\left(\varrho^{-1}(\iota)\right)\right) \sum_{i=1}^{n} q_{i}(\iota)\left(\psi_{1}\left(\phi_{i}(\iota)\right)\right)^{\alpha} \leq 0 \text { for } \iota \geq \iota_{2} .
$$

Since $z(\iota)>0$ and $z^{\prime}(\iota)<0$, there exists a constant $\kappa$ such that

$$
\lim _{\iota \rightarrow \infty} z(\iota)=\kappa<\infty,
$$

where $\kappa \geq 0$. If $\kappa>0$, then there exists $\iota_{3} \geq \iota_{2}$ such that $Q^{-1}\left(\theta_{1}(\iota)\right)>\iota_{2}$ and

$$
z(\iota) \geq \kappa \text { for } \iota \geq \iota_{3}
$$

Integrating (8) from $\iota$ to $\infty$ two times we derive

$$
-z^{\prime}(\iota) \geq \kappa \int_{\iota}^{\infty} \frac{1}{r^{1 / \alpha}(u)}\left(\int_{u}^{\infty} \sum_{i=1}^{n} q_{i}(s)\left(\psi_{1}\left(\phi_{i}(s)\right)\right)^{\alpha}\right)^{1 / \alpha} d u .
$$

Integrating the resulting inequality from $\iota_{3}$ to $l$, we obtain

$$
z\left(\iota_{3}\right) \geq \kappa \int_{\iota_{3}}^{\iota} \int_{v}^{\infty} \frac{1}{r^{1 / \alpha}(u)}\left(\int_{u}^{\infty} \sum_{i=1}^{n} q_{i}(s)\left(\psi_{1}\left(\phi_{i}(s)\right)\right)^{\alpha}\right)^{1 / \alpha} d u d v
$$

which contradicts (4), and so we have $\kappa=0$. Therefore, $\lim _{\iota \rightarrow \infty} z(\iota)=0$. Since $0<x(\iota) \leq z(\iota)$ on $\left[\iota_{1}, \infty\right)$, we obtain $\lim _{\iota \rightarrow \infty} x(\iota)=0$.

Theorem 1. Assume that (2)-(4) hold and $\varrho(\iota) \geq \phi_{i}(\iota)$ for $i=1,2, \cdots, n$. If there exists a function $\zeta \in C^{1}\left(\left[\iota_{0}, \infty\right), \mathbb{R}\right)$ such that

$$
\limsup _{\iota \rightarrow \infty} \int_{T}^{\iota}\left[\zeta(s) \sum_{i=1}^{n} q_{i}(s)\left(\psi_{2}\left(\phi_{i}(s)\right)\right)^{\alpha}\left(\frac{B_{2}\left(\varrho^{-1}\left(\phi_{i}(s)\right), \iota_{2}\right)}{B_{1}\left(s, \iota_{1}\right)}\right)^{\alpha}-\frac{\zeta_{+}^{\prime}(s)}{\left(B_{1}\left(s, \iota_{1}\right)\right)^{\alpha}}\right] d s=\infty,
$$

for all $\iota_{1}, \iota_{2}, T \in\left[\iota_{0}, \infty\right)$, where $T>\iota_{2}>\iota_{1}$, then any solution of (1) is either oscillatory or satisfies $\lim _{l \rightarrow \infty} x(\iota)=0$.

Proof. Assume that (1) has a nonoscillatory solution $x(\iota)$ on $\left[\iota_{0}, \infty\right)$, say there exists $\iota_{1} \in\left[\iota_{0}, \infty\right)$ such that, for $\iota \geq \iota_{1}, x(\iota)>0, x(\varrho(\iota))>0$, and $x\left(\phi_{i}(\iota)\right)>0,(2)$ and (3) hold, and $z(\iota)$ satisfies either $\left(C_{I}\right)$ or $\left(C_{I I}\right)$ for $i=1,2, \cdots, n$. Assuming that $\left(C_{I}\right)$ holds and proceeding as in the proof of Lemma 3, we obtain (5). Since $r(\iota)\left(z^{\prime \prime}(\iota)\right)^{\alpha}$ is decreasing, we see that

$$
z^{\prime}(\iota)=z^{\prime}\left(\iota_{1}\right)+\int_{\iota_{1}}^{\iota} \frac{\left(r(s)\left(z^{\prime \prime}(s)\right)^{\alpha}\right)^{1 / \alpha}}{r^{1 / \alpha}(s)} d s \geq\left(r(\iota)\left(z^{\prime \prime}(\iota)\right)^{\alpha}\right)^{1 / \alpha} B_{1}\left(\iota, \iota_{1}\right) \quad \text { for } \iota \geq \iota_{1} .
$$

From (11), we have for all $\iota \geq \iota_{2}:=\iota_{1}+1$ that

$$
\left(\frac{z^{\prime}(\iota)}{B_{1}\left(\iota, \iota_{1}\right)}\right)^{\prime}=\frac{r^{-1 / \alpha}(\iota)\left[r^{1 / \alpha}(\iota) z^{\prime \prime}(\iota) B_{1}\left(\iota, \iota_{1}\right)-z^{\prime}(\iota)\right]}{\left(B_{1}\left(\iota, \iota_{1}\right)\right)^{2}} \leq 0,
$$


so $z^{\prime}(\iota) / B_{1}\left(\iota, \iota_{1}\right)$ is decreasing for $\iota \geq \iota_{2}$. Next, using the fact that $z^{\prime}(\iota) / B_{1}\left(\iota, \iota_{1}\right)$ is decreasing for $\iota \geq \iota_{2}$, we obtain

$$
\begin{aligned}
z(\iota) & =z\left(\iota_{2}\right)+\int_{\iota_{2}}^{\iota} \frac{z^{\prime}(s)}{B_{1}\left(s, \iota_{1}\right)} B_{1}\left(s, \iota_{1}\right) d s \\
& \geq \frac{z^{\prime}(\iota)}{B_{1}\left(\iota, \iota_{1}\right)} \int_{\iota_{2}}^{\iota} B_{1}\left(s, \iota_{1}\right) d s \\
& =\frac{B_{2}\left(\iota, \iota_{2}\right)}{B_{1}\left(\iota, \iota_{1}\right)} z^{\prime}(\iota) \quad \text { for } \iota \geq \iota_{2} .
\end{aligned}
$$

From (12), for all $\iota \geq \iota_{3}:=\iota_{2}+1$ we have that

$$
\left(\frac{z(\iota)}{B_{2}\left(\iota, \iota_{2}\right)}\right)^{\prime}=\frac{z^{\prime}(\iota) B_{2}\left(\iota, \iota_{2}\right)-z(\iota) B_{1}\left(\iota, \iota_{1}\right)}{\left(B_{2}\left(\iota, \iota_{2}\right)\right)^{2}} \leq 0,
$$

so $z(\iota) / B_{2}\left(\iota, \iota_{2}\right)$ is decreasing for $\iota \geq \iota_{3}$. Next, in view of the fact that $z(\iota) / B_{2}\left(\iota, \iota_{2}\right)$ is decreasing for $\iota \geq \iota_{3}$ and $\varrho(\iota)<\iota$ or $\varrho^{-1}(\iota) \leq \varrho^{-1}\left(\varrho^{-1}(\iota)\right)$, we obtain

$$
\frac{B_{2}\left(\varrho^{-1}\left(\varrho^{-1}(\iota)\right), \iota_{2}\right) z\left(\varrho^{-1}(\iota)\right)}{B_{2}\left(\varrho^{-1}(\iota), \iota_{2}\right)} \geq z\left(\varrho^{-1}\left(\varrho^{-1}(\iota)\right)\right) .
$$

Using (13) in (5) yields

$x(\iota) \geq \frac{1}{p\left(\varrho^{-1}(\iota)\right)}\left[1-\frac{1}{p\left(\varrho^{-1}\left(\varrho^{-1}(\iota)\right)\right)} \frac{B_{2}\left(\varrho^{-1}\left(\varrho^{-1}(\iota)\right), \iota_{2}\right)}{B_{2}\left(\varrho^{-1}(\iota), \iota_{2}\right)}\right] z\left(\varrho^{-1}(\iota)\right)=\psi_{2}(\iota) z\left(\varrho^{-1}(\iota)\right)$,

so

$$
x\left(\phi_{i}(\iota)\right) \geq \psi_{2}\left(\phi_{i}(\iota)\right) z\left(\varrho^{-1}\left(\phi_{i}(\iota)\right)\right), \quad i=1,2, \cdots, n
$$

for $\iota \geq \iota_{3}$. Using (14) in (1) gives

$$
\left(r(\iota)\left(z^{\prime \prime}(\iota)\right)^{\alpha}\right)^{\prime}+\sum_{i=1}^{n} q_{i}(\iota)\left(\psi_{2}\left(\phi_{i}(\iota)\right)\right)^{\alpha} z^{\alpha}\left(\varrho^{-1}\left(\phi_{i}(\iota)\right)\right) \leq 0 .
$$

Next, we define

$$
w(\iota)=\zeta(\iota) \frac{r(\iota)\left(z^{\prime \prime}(\iota)\right)^{\alpha}}{\left(z^{\prime}(\iota)\right)^{\alpha}} \quad \text { for } \iota \geq \iota_{1} .
$$

Then $w(\iota)>0$, and from (15), we see that

$$
\begin{aligned}
w^{\prime}(\iota)= & \frac{\zeta(\iota)}{\left(z^{\prime}(\iota)\right)^{\alpha}}\left[r(\iota)\left(z^{\prime \prime}(\iota)\right)^{\alpha}\right]^{\prime}+\left[\frac{\zeta(\iota)}{\left(z^{\prime}(\iota)\right)^{\alpha}}\right]^{\prime} r(\iota)\left(z^{\prime \prime}(\iota)\right)^{\alpha} \\
= & \zeta^{\prime}(\iota) \frac{r(\iota)\left(z^{\prime \prime}(\iota)\right)^{\alpha}}{\left(z^{\prime}(\iota)\right)^{\alpha}}+\zeta(\iota)\left[\frac{\left(r(\iota)\left(z^{\prime \prime}(\iota)\right)^{\alpha}\right)^{\prime}}{\left(z^{\prime}(\iota)\right)^{\alpha}}-\frac{r(\iota)\left(z^{\prime \prime}(\iota)\right)^{\alpha}\left(\left(z^{\prime}(\iota)\right)^{\alpha}\right)^{\prime}}{\left(z^{\prime}(\iota)\right)^{2 \alpha}}\right] \\
\leq & \zeta_{+}^{\prime}(\iota) \frac{r(\iota)\left(z^{\prime \prime}(\iota)\right)^{\alpha}}{\left(z^{\prime}(\iota)\right)^{\alpha}}-\zeta(\iota)\left[\sum_{i=1}^{n} q_{i}(s)\left(\psi_{2}\left(\phi_{i}(s)\right)\right)^{\alpha} \frac{z^{\alpha}\left(\varrho^{-1}\left(\phi_{i}(\iota)\right)\right)}{\left(z^{\prime}(\iota)\right)^{\alpha}}\right] \\
& -\alpha \zeta(\iota) r(\iota) \frac{\left(z^{\prime \prime}(\iota)\right)^{\alpha+1}}{\left(z^{\prime}(\iota)\right)^{\alpha+1}}
\end{aligned}
$$

for $\iota \geq \iota_{3}$ with $\iota_{3} \in\left(\iota_{2}, \infty\right)$ and $\iota_{2} \in\left(\iota_{1}, \infty\right)$. From (11), $z^{\prime}(\iota)>0$ and $z^{\prime \prime}(\iota)>0,(17)$ yields

$$
w^{\prime}(\iota) \leq \frac{\zeta_{+}^{\prime}(\iota)}{\left(B_{1}\left(\iota, \iota \iota_{1}\right)\right)^{\alpha}}-\zeta(\iota)\left[\sum_{i=1}^{n} q_{i}(s)\left(\psi_{2}\left(\phi_{i}(s)\right)\right)^{\alpha} \frac{z^{\alpha}\left(\varrho^{-1}\left(\phi_{i}(\iota)\right)\right)}{\left(z^{\prime}(\iota)\right)^{\alpha}}\right] \frac{z^{\alpha}(\iota)}{\left(z^{\prime}(\iota)\right)^{\alpha}} \quad \text { for } \iota \geq \iota_{3} .
$$


Using the fact that $z(\iota) / B_{2}\left(\iota, \iota_{2}\right)$ is nonincreasing for $\iota \geq \iota_{3}$, and noting that $\varrho(\iota) \geq \phi_{i}(\iota)$ implies $\varrho^{-1}\left(\phi_{i}(\iota)\right) \leq \iota$, we obtain

$$
\frac{z\left(\varrho^{-1}\left(\phi_{i}(\iota)\right)\right)}{z(\iota)} \geq \frac{B_{2}\left(\varrho^{-1}\left(\phi_{i}(\iota)\right), \iota_{2}\right)}{B_{2}\left(\iota, \iota_{2}\right)}, \quad i=1,2, \cdots, n
$$

for $\iota \geq \iota_{3}$. Substituting (19) and (12) into (18), we obtain

$$
w^{\prime}(\iota) \leq \frac{\zeta_{+}^{\prime}(\iota)}{\left(B_{1}\left(\iota, \iota_{1}\right)\right)^{\alpha}}-\zeta(\iota) \sum_{i=1}^{n} q_{i}(\iota)\left(\psi_{2}\left(\phi_{i}(\iota)\right)\right)^{\alpha}\left(\frac{B_{2}\left(\varrho^{-1}\left(\phi_{i}(\iota)\right), \iota_{2}\right)}{B_{1}\left(\iota, \iota_{1}\right)}\right)^{\alpha} \quad \text { for } \iota \geq \iota_{3} .
$$

An integration of (20) from $\iota_{3}$ to $\iota$ yields

$$
\int_{\iota_{3}}^{\iota}\left[\zeta(s) \sum_{i=1}^{n} q_{i}(s)\left(\psi_{2}\left(\phi_{i}(s)\right)\right)^{\alpha}\left(\frac{B_{2}\left(\varrho^{-1}\left(\phi_{i}(s)\right), \iota_{2}\right)}{B_{1}\left(s, \iota_{1}\right)}\right)^{\alpha}-\frac{\zeta_{+}^{\prime}(s)}{\left(B_{1}\left(s, \iota_{1}\right)\right)^{\alpha}}\right] d s \leq w\left(\iota_{3}\right),
$$

which contradicts (10).

This implies that $\left(C_{I I}\right)$ holds, and so from Lemma 3 , we have $\lim _{\iota \rightarrow \infty} x(\iota)=0$. This completes the proof.

Theorem 2. Assume that (2)-(4) hold and $\varrho(\iota) \geq \phi_{i}(\iota)$ for $i=1,2, \cdots, n$. If there exists a function $\zeta \in C^{1}\left(\left[\iota_{0}, \infty\right), \mathbb{R}\right)$ such that

$$
\limsup _{l \rightarrow \infty} \int_{T}^{l}\left[\zeta(s) \sum_{i=1}^{n} q_{i}(s)\left(\psi_{2}\left(\phi_{i}(s)\right)\right)^{\alpha}\left(\frac{B_{2}\left(e^{-1}\left(\phi_{i}(s)\right), l_{2}\right)}{B_{1}\left(s, l_{1}\right)}\right)^{\alpha}-\frac{r(s)\left(\zeta_{+}^{\prime}(s)\right)^{\alpha+1}}{(\alpha+1)^{\alpha+1} \zeta^{\alpha}(s)}\right] d s=\infty,
$$

for all $\iota_{1}, \iota_{2}, T \in\left[\iota_{0}, \infty\right)$, where $T>\iota_{2}>\iota_{1}$, then any solution of (1) is either oscillatory or satisfies $\lim _{\iota \rightarrow \infty} x(\iota)=0$.

Proof. Assume that (1) has a nonoscillatory solution $x(\iota)$ on $\left[\iota_{0}, \infty\right)$, say there exists $\iota_{1} \in\left[\iota_{0}, \infty\right)$ such that, for $\iota \geq \iota_{1}, x(\iota)>0, x(\varrho(\iota))>0$, and $x\left(\phi_{i}(\iota)\right)>0,(2)$ and (3) hold, for $z(\iota)$ satisfies either $\left(C_{I}\right)$ or $\left(C_{I I}\right)$ and $i=1,2, \cdots, n$. Assume that $\left(C_{I}\right)$ holds. We use the same type of argument as in the proof of the Theorem 1, and arrive at (17). In view of (16), inequality (17) takes the form

$$
w^{\prime}(\iota) \leq \frac{\zeta_{+}^{\prime}(\iota)}{\zeta(\iota)} w(\iota)-\zeta(\iota)\left[\sum_{i=1}^{n} q_{i}(\iota)\left(\psi_{2}\left(\phi_{i}(\iota)\right)\right)^{\alpha} \frac{z^{\alpha}\left(\varrho^{-1}\left(\phi_{i}(\iota)\right)\right)}{\left(z^{\prime}(\iota)\right)^{\alpha}}\right] \frac{z^{\alpha}(\iota)}{\left(z^{\prime}(l)\right)^{\alpha}}-\frac{\alpha w^{(\alpha+1) / \alpha}(\iota)}{(\zeta(\iota) r(\iota))^{1 / \alpha}} .
$$

Using (12) and (19) in (22), for $\iota \geq \iota_{3}$, we obtain

$$
w^{\prime}(\iota) \leq \frac{\zeta_{+}^{\prime}(\iota)}{\zeta(\iota)} w(\iota)-\frac{\alpha w^{(\alpha+1) / \alpha}(\iota)}{(\zeta(\iota) r(\iota))^{1 / \alpha}}-\zeta(\iota) \sum_{i=1}^{n} q_{i}(\iota)\left(\psi_{2}\left(\phi_{i}(\iota)\right)\right)^{\alpha}\left(\frac{B_{2}\left(\varrho^{-1}\left(\phi_{i}(\iota)\right), \iota_{2}\right)}{B_{1}\left(\iota, \iota_{1}\right)}\right)^{\alpha} .
$$

If we apply Lemma 1 with $X=\frac{\alpha^{1 / \lambda}}{\left[(\zeta(\iota) r(\iota))^{1 / \alpha}\right]^{1 / \lambda}} w(\iota), Y=\left[\frac{\alpha}{\alpha+1} \frac{\left[(\zeta(\iota) r(\iota))^{1 / \alpha}\right]^{1 / \lambda}}{\alpha^{1 / \lambda}} \frac{\zeta_{+}^{\prime}(\iota)}{\zeta(\iota)}\right]^{\alpha}$ and $\lambda=\frac{\alpha+1}{\alpha}$, we see that

$$
\frac{\zeta_{+}^{\prime}(\iota)}{\zeta(\iota)} w(\iota)-\frac{\alpha}{(\zeta(\iota) r(\iota))^{1 / \alpha}} w^{(\alpha+1) / \alpha}(\iota) \leq \frac{1}{(\alpha+1)^{\alpha+1}} \frac{r(\iota)\left(\zeta_{+}^{\prime}(\iota)\right)^{\alpha+1}}{\zeta^{\alpha}(\iota)} .
$$

Using this in (23) gives

$$
w^{\prime}(\iota) \leq \frac{1}{(\alpha+1)^{\alpha+1}} \frac{r(\iota)\left(\zeta_{+}^{\prime}(\iota)\right)^{\alpha+1}}{\zeta^{\alpha}(\iota)}-\zeta(\iota) \sum_{i=1}^{n} q_{i}(\iota)\left(\psi_{2}\left(\phi_{i}(\iota)\right)\right)^{\alpha}\left(\frac{B_{2}\left(\varrho^{-1}\left(\phi_{i}(\iota)\right), \iota_{2}\right)}{B_{1}\left(\iota, \iota_{1}\right)}\right)^{\alpha} .
$$


Integrating the latter inequality from $\iota_{3}$ to $\iota$ yields

$\limsup _{\iota \rightarrow \infty} \int_{T}^{\iota}\left[\zeta(s) \sum_{i=1}^{n} q_{i}(s)\left(\psi_{2}\left(\phi_{i}(s)\right)\right)^{\alpha}\left(\frac{B_{2}\left(Q^{-1}\left(\phi_{i}(s)\right), \iota_{2}\right)}{B_{1}\left(s, \iota_{1}\right)}\right)^{\alpha}-\frac{r(s)\left(\zeta_{+}^{\prime}(s)\right)^{\alpha+1}}{(\alpha+1)^{\alpha+1} \zeta^{\alpha}(s)}\right] d s \leq w\left(\iota_{3}\right)$,

which contradicts (21). Therefore $\left(C_{I I}\right)$ holds, and so $\lim _{\iota \rightarrow \infty} x(\iota)=0$ by Lemma 3. This completes the proof.

Next, we examine the oscillation results of solutions of (1) by Philos-type [3]. Let $\mathbb{S}_{0}=\{(\iota, s): a \leq s<\iota<+\infty\}, \mathbb{S}=\{(\iota, s): a \leq s \leq \iota<+\infty\}$; the continuous function $E(\iota, s), E: \mathbb{S} \rightarrow \mathbb{R}$ belongs to the class function $\Re$

$\left(C_{I}\right) E(\iota, \iota)=0$ for $\iota \geq \iota_{0}$ and $E(\iota, s)>0$ for $(\iota, s) \in \mathbb{S}_{0}$,

$\left(C_{I I}\right) \frac{\partial E(\iota, s)}{\partial s} \leq 0,(\iota, s) \in \mathbb{S}_{0}$ and some locally integrable function $e(\iota, s)$ such that

$$
\frac{\partial E(\iota, s)}{\partial s}+E(\iota, s) \frac{\zeta^{\prime}(\iota)}{\zeta(\iota)}=\frac{e_{+}(\iota, s)}{\zeta(\iota)}(E(\iota, s))^{\frac{1}{\alpha}} \quad \text { for all }(\iota, s) \in \mathbb{S}_{0} .
$$

Theorem 3. Assume that (2)-(4) hold and $\varrho(\iota) \geq \phi_{i}(\iota)$ for $i=1,2, \cdots, n$. If there exists a function $\zeta \in C^{1}\left(\left[\iota_{0}, \infty\right), \mathbb{R}\right)$ such that

$$
\begin{aligned}
\limsup _{\iota \rightarrow \infty} \frac{1}{E\left(\iota, \iota_{*}\right)} \int_{\iota_{*}}^{\iota}\left[E(\iota, s) \zeta(s) \sum_{i=1}^{n} q_{i}(s)\left(\psi_{2}\left(\phi_{i}(s)\right)\right)^{\alpha}\right. & \left(\frac{B_{2}\left(\varrho^{-1}\left(\phi_{i}(s)\right), \iota_{2}\right)}{B_{1}\left(s, \iota_{1}\right)}\right)^{\alpha} \\
& \left.-\frac{r(s)\left(\zeta_{+}^{\prime}(s)\right)^{\alpha+1}}{(\alpha+1)^{\alpha+1} \zeta^{\alpha}(s)}\right] d s=\infty,
\end{aligned}
$$

for all $\iota_{1}, \iota_{2}, \iota_{*} \in\left[\iota_{0}, \infty\right)$, where $\iota_{*}>\iota_{2}>\iota_{1}$, then any solution of (1) is either oscillatory or satisfies $\lim _{\iota \rightarrow \infty} x(\iota)=0$.

Proof. Assume that (1) has a nonoscillatory solution $x(\iota)$ on $\left[\iota_{0}, \infty\right)$, say there exists $\iota_{1} \in\left[\iota_{0}, \infty\right)$ such that, for $\iota \geq \iota_{1}, x(\iota)>0, x(\varrho(\iota))>0$, and $x\left(\phi_{i}(\iota)\right)>0,(2)$ and (3) hold, for $z(\iota)$ satisfies either $\left(C_{I}\right)$ or $\left(C_{I I}\right)$ and $i=1,2, \cdots, n$. Assume that $\left(C_{I}\right)$ holds. Following the same arguments as in the proof of the Theorem 1, we arrive at (17). In view of (16), inequality (17) takes the form

$$
\zeta(\iota) \sum_{i=1}^{n} q_{i}(\iota)\left(\psi_{2}\left(\phi_{i}(\iota)\right)\right)^{\alpha}\left(\frac{B_{2}\left(e^{-1}\left(\phi_{i}(\iota)\right), \iota_{2}\right)}{B_{1}\left(\iota, l_{1}\right)}\right)^{\alpha} \leq-w^{\prime}(\iota)+\frac{\zeta_{+}^{\prime}(\iota)}{\zeta(\iota)} w(\iota)-\frac{\alpha w^{(\alpha+1) / \alpha}(\iota)}{(\zeta(\iota) r(\iota))^{1 / \alpha}} .
$$

Multiplying by $E(\iota, s)$ and integrating (25) from $\iota_{3}$ to $\iota$, one can obtain that

$$
\begin{aligned}
& \int_{\iota_{3}}^{\iota} E(\iota, s) \zeta(s) \sum_{i=1}^{n} q_{i}(s)\left(\psi_{2}\left(\phi_{i}(s)\right)\right)^{\alpha}\left(\frac{B_{2}\left(\varrho^{-1}\left(\phi_{i}(s)\right), \iota_{2}\right)}{B_{1}\left(s, \iota_{1}\right)}\right)^{\alpha} d s \\
& \leq-\int_{\iota_{3}}^{\iota} E(\iota, s) w^{\prime}(s) d s+\int_{\iota_{3}}^{\iota} E(\iota, s) \frac{\zeta_{+}^{\prime}(s)}{\zeta(s)} w(s) d s-\int_{\iota_{3}}^{\iota} E(\iota, s) \frac{\alpha w^{(\alpha+1) / \alpha}(s)}{(\zeta(s) r(s))^{1 / \alpha}} d s \\
& \leq E\left(\iota, \iota_{3}\right) w\left(\iota_{3}\right)+\int_{\iota_{3}}^{\iota}\left\{\frac{\partial E(\iota, s)}{\partial s}+E(\iota, s) \frac{\zeta_{+}^{\prime}(s)}{\zeta(s)}\right\} w(s) d s-\int_{\iota_{3}}^{\iota} E(\iota, s) \frac{\alpha w^{(\alpha+1) / \alpha}(s)}{(\zeta(s) r(s))^{1 / \alpha}} d s \\
& \leq E\left(\iota, \iota_{3}\right) w\left(\iota_{3}\right)+\int_{\iota_{3}}^{\iota}(E(\iota, s))^{\frac{1}{\alpha}} \frac{e_{+}(\iota, s)}{\zeta(s)} w(s) d s-\int_{\iota_{3}}^{\iota} E(\iota, s) \frac{\alpha w^{(\alpha+1) / \alpha}(s)}{(\zeta(s) r(s))^{1 / \alpha}} d s .
\end{aligned}
$$

Now, using the Lemma 1 , set

$$
X=\left[\frac{\alpha E(l, s)}{(\zeta(s) r(s))^{1 / \alpha}}\right]^{1 / \lambda} w(s)
$$


and

$$
Y=\left[\frac{\alpha}{1+\alpha}\left[\frac{\left((\zeta(s) r(s))^{1 / \alpha}\right)^{1 / \lambda}}{\alpha^{1 / \lambda}} \frac{e_{+}(l, s)}{\zeta(s)}\right]^{\alpha}\right.
$$

we obtain that

$$
\int_{\iota_{3}}^{\iota}\left[E(\iota, s) \zeta(s) \sum_{i=1}^{n} q_{i}(s)\left(\psi_{2}\left(\phi_{i}(s)\right)\right)^{\alpha}\left(\frac{B_{2}\left(e^{-1}\left(\phi_{i}(s)\right), \iota_{2}\right)}{B_{1}\left(s, l_{1}\right)}\right)^{\alpha}-\frac{r(s)\left(\zeta_{+}^{\prime}(s)\right)^{\alpha+1}}{(\alpha+1)^{\alpha+1} \zeta^{\alpha}(s)}\right] d s \leq E\left(\iota, \iota_{3}\right) w\left(\iota_{3}\right),
$$

which contradicts (24). Therefore $\left(C_{I I}\right)$ holds, and so $\lim _{\iota \rightarrow \infty} x(\iota)=0$ by Lemma 3. This completes the proof.

Corollary 1. Suppose that all conditions of Theorem 3 are satisfied with (24) replaced by

$$
\limsup _{\iota \rightarrow \infty} \frac{1}{E\left(\iota, \iota_{*}\right)} \int_{\iota_{*}}^{\iota} E(\iota, s) \zeta(s) \sum_{i=1}^{n} q_{i}(s)\left(\psi_{2}\left(\phi_{i}(s)\right)\right)^{\alpha}\left(\frac{B_{2}\left(\varrho^{-1}\left(\phi_{i}(s)\right), \iota_{2}\right)}{B_{1}\left(s, \iota_{1}\right)}\right)^{\alpha} d s=\infty
$$

and

$$
\limsup _{l \rightarrow \infty} \frac{1}{E\left(\iota, l_{*}\right)} \int_{l_{*}}^{\iota} \frac{r(s)\left(\zeta_{+}^{\prime}(s)\right)^{\alpha+1}}{\zeta^{\alpha}(s)} d s<\infty,
$$

then any solution of (1) is either oscillatory or satisfies $\lim _{\iota \rightarrow \infty} x(\iota)=0$.

Theorem 4. Let $\alpha \geq 1$. Assume that (2)-(4) hold and $\varrho(\iota) \geq \phi_{i}(\iota)$ for $i=1,2, \cdots, n$. If there exists a function $\zeta \in C^{1}\left(\left[\iota_{0}, \infty\right), \mathbb{R}\right)$ such that

$$
\limsup _{\iota \rightarrow \infty} \int_{T}^{\iota}\left[\zeta(s) \sum_{i=1}^{n} q_{i}(s)\left(\psi_{2}\left(\phi_{i}(s)\right)\right)^{\alpha}\left(\frac{B_{2}\left(\varrho^{-1}\left(\phi_{i}(s)\right), \iota_{2}\right)}{B_{1}\left(s, \iota_{1}\right)}\right)^{\alpha}-\frac{r^{1 / \alpha}(s)\left(\zeta_{+}^{\prime}(s)\right)^{2}}{4 \alpha \zeta(s)\left[B_{1}\left(s, \iota_{1}\right)\right]^{\alpha-1}}\right] d s=\infty,
$$

for all $\iota_{1}, \iota_{2}, T \in\left[\iota_{0}, \infty\right)$, where $T>\iota_{2}>\iota_{1}$, then any solution of (1) is either oscillatory or satisfies $\lim _{\iota \rightarrow \infty} x(\iota)=0$.

Proof. Let (1) have a nonoscillatory solution $x(\iota)$ on $\left[\iota_{0}, \infty\right)$, and say there exists $\iota_{1} \in\left[\iota_{0}, \infty\right)$ such that, for $\iota \geq \iota_{1}, x(\iota)>0, x(\varrho(\iota))>0$, and $x\left(\phi_{i}(\iota)\right)>0$, (2) and (3) hold, and $z(\iota)$ satisfies either $\left(C_{I}\right)$ or $\left(C_{I I}\right)$ for $i=1,2, \cdots, n$. Assume $\left(C_{I}\right)$ holds. Following the same arguments as in the proof of the Theorem 2, we arrive at (23), which can be rewritten as

$$
w^{\prime}(\iota) \leq \frac{\zeta_{+}^{\prime}(\iota)}{\zeta(\iota)} w(\iota)-\zeta(\iota) \Omega_{2}(\iota)\left(\frac{B_{2}\left(\varrho^{-1}((\iota)), \iota_{2}\right)}{B_{1}\left(\iota, \iota_{1}\right)}\right)^{\alpha}-\frac{\alpha w^{2}(\iota) w^{\frac{1}{\alpha}-1}(\iota)}{(\zeta(\iota) r(\iota))^{1 / \alpha}} .
$$

From (11) and (16), we see that

$$
\begin{aligned}
w^{\frac{1}{\alpha}-1}(\iota) & =(\zeta(\iota) r(\iota))^{\frac{1}{\alpha}-1} \frac{\left(z^{\prime \prime}(\iota)\right)^{1-\alpha}}{\left(z^{\prime}(\iota)\right)^{1-\alpha}} \\
& =(\zeta(\iota) r(\iota))^{\frac{1}{\alpha}-1}\left(\frac{z^{\prime}(\iota)}{z^{\prime \prime}(\iota)}\right)^{\alpha-1} \\
& \geq(\zeta(\iota) r(\iota))^{\frac{1}{\alpha}-1}\left[r^{1 / \alpha}(\iota) B_{1}\left(\iota, \iota_{1}\right)\right]^{\alpha-1} \\
& =\zeta^{\frac{1}{\alpha}-1}(\iota)\left[B_{1}\left(\iota, \iota_{1}\right)\right]^{\alpha-1} .
\end{aligned}
$$

Using (29) in (28), for $\iota \geq \iota_{3}$, we obtain

$$
w^{\prime}(\iota) \leq-\zeta(\iota) \sum_{i=1}^{n} q_{i}(\iota)\left(\psi_{2}\left(\phi_{i}(\iota)\right)\right)^{\alpha}\left(\frac{B_{2}\left(\varrho^{-1}\left(\phi_{i}(\iota)\right), \iota_{2}\right)}{B_{1}\left(l, l_{1}\right)}\right)^{\alpha}+\frac{\zeta_{+}^{\prime}(\iota)}{\zeta(\iota)} w(\iota)-\frac{\alpha\left[B_{1}\left(\iota, l_{1}\right)\right]^{\alpha-1}}{\zeta(\iota) r^{1 / \alpha}(\iota)} w^{2}(\iota) .
$$


Bringing the square to a close with respect to $w$, from (30) it follows that

$$
w^{\prime}(\iota) \leq-\zeta(\iota) \sum_{i=1}^{n} q_{i}(\iota)\left(\psi_{2}\left(\phi_{i}(\iota)\right)\right)^{\alpha}\left(\frac{B_{2}\left(\varrho^{-1}\left(\phi_{i}(\iota)\right), \iota_{2}\right)}{B_{1}\left(\iota, \iota_{1}\right)}\right)^{\alpha}+\frac{r^{1 / \alpha}(\iota)}{4 \alpha\left[B_{1}(\iota, \iota l)\right]^{\alpha-1}} \frac{\left(\zeta_{+}^{\prime}(\iota)\right)^{2}}{\zeta(\iota)} .
$$

Integrating this inequality from $\iota_{3}$ to $\iota$ gives

$$
\int_{T}^{\iota}\left[\zeta(s) \sum_{i=1}^{n} q_{i}(s)\left(\psi_{2}\left(\phi_{i}(s)\right)\right)^{\alpha}\left(\frac{B_{2}\left(\varrho^{-1}\left(\theta_{2}(s)\right), \iota_{2}\right)}{B_{1}\left(s, \iota_{1}\right)}\right)^{\alpha}-\frac{r^{1 / \alpha}(s)\left(\zeta_{+}^{\prime}(s)\right)^{2}}{4 \alpha \zeta(s)\left[B_{1}\left(s, \iota_{1}\right)\right]^{\alpha-1}}\right] d s \leq w\left(\iota_{3}\right),
$$

which contradicts (27).

If $\left(C_{I I}\right)$ holds, then again from Lemma 3, we have $\lim _{l \rightarrow \infty} x(\iota)=0$. The proof is complete.

Next, we give oscillation results in the case when $\varrho(\iota) \leq \phi_{i}(\iota)$ for $i=1,2, \cdots, n$ holds.

Theorem 5. Assume that (2)-(4) hold and $\varrho(\iota) \leq \phi_{i}(\iota)$ for $i=1,2, \cdots, n$. If there exists a function $\zeta \in C^{1}\left(\left[\iota_{0}, \infty\right), \mathbb{R}\right)$ such that

$$
\limsup _{\iota \rightarrow \infty} \int_{T}^{\iota}\left[\zeta(s) \Omega_{2}(s)\left(\frac{B_{2}\left(s, \iota_{2}\right)}{B_{1}\left(s, \iota_{1}\right)}\right)^{\alpha}-\frac{\zeta_{+}^{\prime}(s)}{\left(B_{1}\left(s, \iota_{1}\right)\right)^{\alpha}}\right] d s=\infty,
$$

for all $\iota_{1}, \iota_{2}, T \in\left[\iota_{0}, \infty\right)$, where $T>\iota_{2}>\iota_{1}$, then any solution of $(1)$ is either oscillatory or satisfies $\lim _{\iota \rightarrow \infty} x(\iota)=0$.

Proof. Let (1) has a nonoscillatory solution $x(\iota)$ on $\left[\iota_{0}, \infty\right)$, say there exists $\iota_{1} \in\left[\iota_{0}, \infty\right)$ such that, for $\iota \geq \iota_{1}, x(\iota)>0, x(\varrho(\iota))>0$, and $x\left(\phi_{i}(\iota)\right)>0$, (2) and (3) hold, for $z(\iota)$ satisfies either $\left(C_{I}\right)$ or $\left(C_{I I}\right)$ and $i=1,2, \cdots, n$. Assume that $\left(C_{I}\right)$ holds. Following the same arguments as in the proof of the Theorem 1, we arrive at (18). Using the fact that $\varrho(\iota)$ is strictly increasing and $\varrho(\iota) \leq \phi_{i}(\iota)$, we have

$$
\iota \leq \varrho^{-1}\left(\phi_{i}(\iota)\right), \quad i=1,2, \cdots, n .
$$

Thus, in view of the fact that $z(\iota)$ is increasing, we obtain

$$
\frac{z\left(\varrho^{-1}\left(\phi_{i}(\iota)\right)\right)}{z(\iota)} \geq 1, \quad i=1,2, \cdots, n .
$$

Using (32) in (18), we obtain that

$$
w^{\prime}(\iota) \leq \frac{\zeta_{+}^{\prime}(\iota)}{\left(B_{1}\left(\iota, \iota_{1}\right)\right)^{\alpha}}-\zeta(\iota) \frac{z^{\alpha}(\iota)}{\left(z^{\prime}(\iota)\right)^{\alpha}} \sum_{i=1}^{n} q_{i}(s)\left(\psi_{2}\left(\phi_{i}(s)\right)\right)^{\alpha} \quad \text { for } \iota \geq \iota_{3} .
$$

In view of (12), (33) takes the form

$$
w^{\prime}(\iota) \leq \frac{\zeta_{+}^{\prime}(\iota)}{\left(B_{1}\left(\iota, \iota_{1}\right)\right)^{\alpha}}-\zeta(\iota) \Omega_{2}(\iota)\left(\frac{B_{2}\left(\iota, \iota_{2}\right)}{B_{1}\left(\iota, \iota_{1}\right)}\right)^{\alpha} \quad \text { for } \iota \geq \iota_{3} .
$$

The remainder of the proof is similar to that of Theorem 1 and so we omit it.

Theorem 6. Assume that (2)-(4) hold and $\varrho(\iota) \leq \phi_{i}(\iota)$ for $i=1,2, \cdots, n$. If there exists a function $\zeta \in C^{1}\left(\left[\iota_{0}, \infty\right), \mathbb{R}\right)$ such that

$$
\limsup _{\iota \rightarrow \infty} \int_{T}^{\iota}\left[\zeta(s) \Omega_{2}(s)\left(\frac{B_{2}\left(s, \iota_{2}\right)}{B_{1}\left(s, \iota_{1}\right)}\right)^{\alpha}-\frac{r(s)\left(\zeta_{+}^{\prime}(s)\right)^{\alpha+1}}{(\alpha+1)^{\alpha+1} \zeta^{\alpha}(s)}\right] d s=\infty,
$$


for all $\iota_{1}, \iota_{2}, T \in\left[\iota_{0}, \infty\right)$, where $T>\iota_{2}>\iota_{1}$, then any solution of (1) is either oscillatory or satisfies $\lim _{\iota \rightarrow \infty} x(\iota)=0$.

Theorem 7. Assume that (2)-(4) hold and $\varrho(\iota) \leq \phi_{i}(\iota)$ for $i=1,2, \cdots, n$. If there exists a function $\zeta \in C^{1}\left(\left[\iota_{0}, \infty\right), \mathbb{R}\right)$ such that

$$
\limsup _{\iota \rightarrow \infty} \frac{1}{E\left(\iota, \iota_{*}\right)} \int_{\iota_{*}}^{\iota}\left[E(\iota, s) \zeta(s) \Omega_{2}(s)\left(\frac{B_{2}\left(s, \iota_{2}\right)}{B_{1}\left(s, \iota_{1}\right)}\right)^{\alpha}-\frac{r(s)\left(\zeta_{+}^{\prime}(s)\right)^{\alpha+1}}{(\alpha+1)^{\alpha+1} \zeta^{\alpha}(s)}\right] d s=\infty,
$$

for all $\iota_{1}, \iota_{2}, \iota_{*} \in\left[\iota_{0}, \infty\right)$, where $\iota_{*}>\iota_{2}>\iota_{1}$, then any solution of (1) is either oscillatory or satisfies $\lim _{\iota \rightarrow \infty} x(\iota)=0$.

Corollary 2. Suppose that all conditions of Theorem 7 are satisfied with (36) replaced by

$$
\limsup _{\iota \rightarrow \infty} \frac{1}{E\left(l, \iota_{*}\right)} \int_{\iota_{*}}^{\iota} E(\iota, s) \zeta(s) \Omega_{2}(s)\left(\frac{B_{2}\left(s, \iota_{2}\right)}{B_{1}\left(s, \iota_{1}\right)}\right)^{\alpha} d s=\infty
$$

and

$$
\limsup _{l \rightarrow \infty} \frac{1}{E\left(\iota, \iota_{*}\right)} \int_{\iota_{*}}^{\iota} \frac{r(s)\left(\zeta_{+}^{\prime}(s)\right)^{\alpha+1}}{(\alpha+1)^{\alpha+1} \zeta^{\alpha}(s)} d s<\infty,
$$

then any solution of (1) is either oscillatory or satisfies $\lim _{\iota \rightarrow \infty} x(\iota)=0$.

Theorem 8. Let $\alpha \geq 1$. Assume that (2)-(4) hold and $\varrho(\iota) \leq \phi_{i}(\iota)$ for $i=1,2, \cdots, n$. If there exists a function $\zeta \in C^{1}\left(\left[\iota_{0}, \infty\right), \mathbb{R}\right)$ such that

$$
\limsup _{\iota \rightarrow \infty} \int_{T}^{\iota}\left[\zeta(s) \Omega_{2}(s)\left(\frac{B_{2}\left(s, \iota_{2}\right)}{B_{1}\left(s, \iota_{1}\right)}\right)^{\alpha}-\frac{r^{1 / \alpha}(s)}{4 \alpha\left[B_{1}\left(s, \iota_{1}\right)\right]^{\alpha-1}} \frac{\left(\zeta_{+}^{\prime}(s)\right)^{2}}{\zeta(s)}\right] d s=\infty,
$$

for all $\iota_{1}, \iota_{2}, T \in\left[\iota_{0}, \infty\right)$, where $T>\iota_{2}>\iota_{1}$, then any solution of (1) is either oscillatory or satisfies $\lim _{\iota \rightarrow \infty} x(\iota)=0$.

Example 1. Consider the differential equation

$$
\left(\left((x(\iota)+8 x(\iota / 2))^{\prime \prime}\right)^{3}\right)^{\prime}+\iota^{2} x^{3}(\iota / 4)+\iota^{3} x^{3}(\iota / 8)=0, \quad \iota \geq 1
$$

where $\alpha=3, r(\iota)=1, p(\iota)=8, \varrho(\iota)=\iota / 2, q_{1}(\iota)=\iota^{2}, q_{2}(\iota)=\iota^{3}, \phi_{1}(\iota)=\iota / 4$ and $\phi_{2}(\iota)=\iota / 8$. Then, we obtain

$$
\begin{aligned}
& B_{1}\left(\iota, \iota_{1}\right)=B_{1}(\iota, 1)=\iota-1, \\
& B_{2}\left(\iota, \iota_{2}\right)=B_{2}(\iota, 2)=\left(\iota^{2}-2 \iota\right) / 2, \\
& B_{2}\left(\varrho^{-1}(\iota), \iota_{2}\right)=B_{2}(2 \iota, 2)=2 \iota^{2}-2 \iota, \\
& B_{2}\left(\varrho^{-1}\left(\varrho^{-1}(\iota)\right), \iota_{2}\right)=B_{2}(4 \iota, 2)=8 \iota^{2}-4 \iota \\
& B_{2}\left(\varrho^{-1}\left(\phi_{1}(\iota)\right), \iota_{2}\right)=B_{2}(\iota / 2,2)=\frac{4 \iota^{2}-\iota}{8} \\
& B_{2}\left(\varrho^{-1}\left(\phi_{2}(\iota)\right), \iota_{2}\right)=B_{2}(\iota / 4,2)=\frac{8 \iota^{2}-\iota}{32},
\end{aligned}
$$


and

$$
\begin{aligned}
& \psi_{1}(\iota)=\frac{1}{8}\left(1-\frac{1}{8}\right)=7 / 64>0 \\
& \psi_{2}(\iota)=\frac{1}{8}\left(1-\frac{1}{8} \frac{8 \iota^{2}-4 \iota}{2 \iota^{2}-2 \iota}\right)=\frac{1}{8}\left(\frac{2 \iota-1}{\iota-1}\right) \geq \frac{1}{32}>0, \quad \text { for } \iota \geq \iota_{2}=2, \\
& \Omega_{1}(\iota)=\sum_{i=1}^{2} q_{i}(\iota)\left(\psi_{1}\left(\phi_{i}(\iota)\right)\right)^{\alpha}=\iota^{2}\left(\frac{7}{64}\right)^{3}+\iota^{3}\left(\frac{7}{64}\right)^{3}=\iota^{2}\left(\frac{7}{64}\right)^{3}(1+\iota) .
\end{aligned}
$$

It is easy to verify that

$$
\int_{\iota_{0}}^{\infty} \int_{v}^{\infty} \frac{1}{r^{1 / \alpha}(u)}\left(\int_{u}^{\infty} \Omega_{1}(s) d s\right)^{1 / \alpha} d u d v=\int_{1}^{\infty} \int_{v}^{\infty} \int_{u}^{\infty}\left(\frac{7}{64}\right)\left(s^{2}(1+s)\right)^{\frac{1}{3}} d s d u d v=\infty,
$$

and picking $\zeta(\iota)=\iota$, we see that

$$
\begin{array}{r}
\int_{T}^{\infty}\left[s \sum_{i=1}^{2} q_{i}(s)\left(\psi_{2}\left(\phi_{i}(s)\right)\right)^{3}\left(\frac{B_{2}\left(\varrho^{-1}\left(\phi_{i}(s)\right), \iota_{2}\right)}{B_{1}\left(s, \iota_{1}\right)}\right)^{3}-\frac{1}{\left(B_{1}\left(s, \iota_{1}\right)\right)^{3}}\right] d s \\
=\int_{2}^{\infty}\left\{s^{3}\left(\frac{7}{64}\right)^{3}\left(\frac{4 s^{2}-s}{8(s-1)}\right)^{3}+s^{4}\left(\frac{1}{32}\right)^{3}\left(\frac{8 s^{2}-s}{32(s-1)}\right)^{3}-\frac{1}{(s-1)^{3}}\right\} d s=\infty .
\end{array}
$$

Hence, any solution of (38) is either oscillatory or satisfies $\lim _{\iota \rightarrow \infty} x(\iota)=0$ by Theorem 1 .

Example 2. Consider the differential equation

$$
\left(\left(\left(x(\iota)+\frac{7 \iota+8}{\iota+1} x(\iota-2)\right)^{\prime \prime}\right)^{1 / 5}\right)^{\prime}+\left(\iota^{2}+\iota\right) x^{1 / 5}\left(\iota-\frac{3}{2}\right)+\left(\iota^{3}+\iota\right) x^{1 / 5}\left(\iota-\frac{1}{2}\right)=0, \quad \iota \geq 2
$$

where $\alpha=1 / 5, r(\iota)=1, p(\iota)=\frac{7 \imath+8}{\iota+1}, \varrho(\iota)=\iota-2, q_{1}(\iota)=\iota^{2}+\iota, q_{2}(\iota)=\iota^{3}+\iota, \phi_{1}(\iota)=$ $\iota-3 / 2$ and $\phi_{2}(\iota)=\iota-1 / 2$. Then, we obtain

$$
\begin{aligned}
& 7 \leq p(\iota)<8 \\
& B_{1}\left(\iota, \iota_{1}\right)=B_{1}(\iota, 2)=\iota-2 \\
& B_{2}\left(\iota, \iota_{2}\right)=B_{2}(\iota, 3)=\left(\iota^{2}-4 \iota+3\right) / 2 \\
& B_{2}\left(\varrho^{-1}(\iota), \iota_{2}\right)=B_{2}(\iota+2,3)=\left(\iota^{2}-1\right) / 2 \\
& B_{2}\left(\varrho^{-1}\left(\varrho^{-1}(\iota)\right), \iota_{2}\right)=B_{2}(\iota+4,3)=\left(\iota^{2}+4 \iota+3\right) / 2 \iota
\end{aligned}
$$

and

$$
\begin{aligned}
& \psi_{1}(\iota) \geq \frac{1}{8}\left(1-\frac{1}{7}\right)=3 / 28>0 \\
& \psi_{2}(\iota)=\frac{1}{8}\left(1-\frac{1}{7} \frac{\iota^{2}+4 \iota+3}{\iota^{2}-1}\right) \geq \frac{1}{14}>0, \quad \text { for } \iota \geq \iota_{2}=3, \\
& \Omega_{1}(\iota)=\sum_{i=1}^{2} q_{i}(\iota)\left(\psi_{1}\left(\phi_{i}(\iota)\right)\right)^{\frac{1}{5}}=\left(\frac{3}{28}\right)^{\frac{1}{5}}\left(\iota^{3}+\iota^{2}+2 \iota\right), \\
& \Omega_{2}(\iota)=\sum_{i=1}^{2} q_{i}(\iota)\left(\psi_{2}\left(\phi_{i}(\iota)\right)\right)^{\alpha} \geq\left(\frac{1}{14}\right)^{1 / 5}\left(\iota^{3}+\iota^{2}+2 \iota\right), \quad \text { for } \iota \geq \iota_{2}=3 .
\end{aligned}
$$


It is easy to verify that

$$
\begin{array}{r}
\int_{\iota_{0}}^{\infty} \int_{v}^{\infty} \frac{1}{r^{1 / \alpha}(u)}\left(\int_{u}^{\infty} \Omega_{1}(s) d s\right)^{1 / \alpha} d u d v= \\
\left(\frac{3}{28}\right)^{\frac{1}{5}} \int_{2}^{\infty} \int_{v}^{\infty} \int_{u}^{\infty}\left(s^{3}+s^{2}+2 s\right)^{1 / 5} d s d u d v=\infty,
\end{array}
$$

and picking $\zeta(\iota)=1$, we see that

$$
\begin{aligned}
& \int_{T}^{\infty}\left[\zeta(s) \Omega_{2}(s)\right.\left.\left(\frac{B_{2}\left(s, \iota_{2}\right)}{B_{1}\left(s, \iota_{1}\right)}\right)^{1 / 5}-\frac{\zeta_{+}^{\prime}(s)}{\left(B_{1}\left(s, \iota_{1}\right)\right)^{1 / 5}}\right] d s \\
&=\int_{3}^{\infty}\left\{\left(\frac{1}{14}\right)^{1 / 5}\left(s^{3}+s^{2}+2 s\right)\left(\frac{s^{2}-4 s+3}{2 s-4}\right)^{1 / 5}\right\} d s=\infty .
\end{aligned}
$$

Hence, any solution of (39) is either oscillatory or satisfies $\lim _{\iota \rightarrow \infty} x(\iota)=0$, by Theorem 5 .

\section{Conclusions}

We established several oscillation theorems for (1) under the assumptions of $\varrho(\iota) \geq \phi_{i}(\iota)$ and $\varrho(\iota) \leq \phi_{i}(\iota)$ for $i=1,2, \cdots, n$, when $p(\iota) \geq 1$. The main outcomes were proven via the means of a generalized Riccati technique, integral averaging conditions under the assumptions of $\int_{L_{0}}^{\infty} r^{-1 / \alpha}(s) d s=\infty$. Two examples were given to prove the significance of new theorems. The primary conclusions given in this work are basically new and have a high degree of generality. For future consideration, it will be of great importance to study the oscillation of (1) when $\int_{\iota_{0}}^{\infty} r^{-1 / \alpha}(s) d s<\infty$.

Author Contributions: Conceptualization, M.S.K., R.E., V.G., O.B., K.A.-S. and K.N.; methodology, M.S.K., R.E., V.G., O.B., K.A.-S. and K.N.; investigation, M.S.K., R.E., V.G., O.B., K.A.-S. and K.N.; resources, M.S.K., R.E., V.G., O.B., K.A.-S. and K.N.; data curation, M.S.K., R.E., V.G., O.B., K.A.-S. and K.N.; writing—original draft preparation, M.S.K., R.E., V.G., O.B., K.A.-S. and K.N.; writing-review and editing, M.S.K., R.E., V.G., O.B., K.A.-S. and K.N.; supervision, M.S.K., R.E., V.G., O.B., K.A.-S. and K.N.; project administration, M.S.K., R.E., V.G., O.B., K.A.-S. and K.N.; funding acquisition, M.S.K., R.E., V.G., O.B., K.A.-S. and K.N. All authors read and agreed to the published version of the manuscript.

Funding: This research received no external funding.

Institutional Review Board Statement: Not applicable.

Informed Consent Statement: Not applicable.

Data Availability Statement: Not applicable.

Conflicts of Interest: The authors declare no conflict of interest.

\section{References}

1. Hale, J.K. Theory of Functional Differential Equations; Springer: New York, NY, USA, 1977.

2. Agarwal, R.P.; Bazighifan, O.; Ragusa, M.A. Nonlinear Neutral Delay Differential Equations of Fourth-Order: Oscillation of Solutions. Entropy 2021, 23, 129. [CrossRef] [PubMed]

3. Philos, C.G. Oscillation theorems for linear differential equations of second order. Arch. Der Math. 1989, 53, 482-492. [CrossRef]

4. Bazighifan, O.; Mofarreh, F.; Nonlaopon, K. On the Qualitative Behavior of Third-Order Differential Equations with a Neutral Term. Symmetry 2021, 13, 1287. [CrossRef]

5. Elayaraja, R.; Sathish Kumar, M.; Ganesan, V. Nonexistence of Kneser solution for third order nonlinear neutral delay differential equations. J. Phys. Conf. Ser. 2021, 1850, 012054. [CrossRef]

6. Chen, D.X.; Liu, J.C. Asymptotic behavior and oscilation of solutions of third-order nonlinear neutral delay dynamic equations on time scales. Can. Appl. Math. Q. 2008, 16, 19-43.

7. Graef, J.R.; Savithri, R.; Thandapani, E. Oscillatory properties of third order neutral delay differential equations. In Proceedings of the Fourth International Conference on Dynamical Systems and Differential Equations, Wilmington, NC, USA, 24-27 May 2002; pp. 342-350. 
8. Graef, J.R.; Saker, S.H. Oscillation theory of third-order nonlinear functional differential equations. Hiroshima Math. J. 2013, 43, 49-72. [CrossRef]

9. Chatzarakis, G.E.; Grace, S.R.; Jadlovská, I.; Li, T.; Tunç, E. Oscillation criteria for third-order Emden-Fowler differential equations with unbounded neutral coefficients. Complexity 2019, 2019, 5691758. [CrossRef]

10. Sathish Kumar, M.; Ganesan, V. Asymptotic behavior of solutions of third-order neutral differential equations with discrete and distributed delay. AIMS Math. 2020, 5, 3851-3874. [CrossRef]

11. Sathish Kumar, M.; Bazighifan, O.; Almutairi, A.; Chalishajar, D.N. Philos-type oscillation results for third-order differential equation with mixed neutral terms. Mathematics 2021, 9, 1021. [CrossRef]

12. Ganesan, V.; Sathish Kumar, M. On the oscillation of a third order nonlinear differential equations with neutral type. Ural Math. J. 2017, 3, 122-129. [CrossRef]

13. Sathish Kumar, M.; Janaki, S.; Ganesan, V. Some new oscillatory behavior of certain third-order nonlinear neutral differential equations of mixed type. Int. J. Appl. Comput. Math. 2018, 78, 1-14. [CrossRef]

14. Tunc, E.; Grace, S.R. Oscillatory behavior of solutions to third-order nonlinear differential equations with a superlinear neutral term. Electron. J. Differ. Equ. 2020, 2020, 1-11.

15. Qin, G.; Huang, C.; Xie, Y.; Wen, F. Asymptotic behavior for third-order quasi-linear differential equations. Adv. Differ. Equ. 2013, 2013, 305. [CrossRef]

16. AlGhamdi, A.; Bazighifan, O.; El-Nabulsi, R.A. Important Criteria for Asymptotic Properties of Nonlinear Differential Equations. Mathematics 2021, 9, 1659. [CrossRef]

17. Baculíková, B.; Dzurina, J. Oscillation of third-order neutral differential equations. Math. Comput. Model. 2010, 52, 215-226. [CrossRef]

18. Dzurina, J.; Thandapani, E.; Tamilvanan, S. Oscillation of solutions to third-order half-linear neutral differential equations. Electron. J. Differ. Equ. 2012, 29, 1-9.

19. Li, T.; Thandapani, E. Oscillation theorems for odd-order neutral differential equations. Funct. Differ. Equ. 2012, 19, 147-155.

20. Jiang, Y.; Jiang, C.; Li, T. Oscillatory behavior of third-order nonlinear neutral delay differential equations. Adv. Differ. Equ. 2016, 171, 171. [CrossRef]

21. Tunc, E. Oscillatory and asymptotic behavior of third-order neutral differential equations with distributed deviating arguments. Electron. J. Differ. Equ. 2017, 2017, 267. [CrossRef]

22. Soliman, A.A.; Sallam, R.A.; Elbitar, A.; Hassan, A.M. Oscillation criteria of third order nonlinear neutral differential equations. Int. J. Appl. Math. Res. 2012, 1, 268-281. [CrossRef]

23. Hardy, G.H.; Littlewood, J.E.; Polya, G. Inequalities, 2nd ed.; Cambridge University Press: Cambridge, UK, 1952. 